\title{
A note on the Taylor's decomposition on four points for a third-order differential equation
}

\author{
Allaberen Ashyralyev ${ }^{1}$ and Doghonay Arjmand ${ }^{2}$ \\ ${ }^{1}$ Dep. of Math., Fatih University, Istanbul, Turkey \\ ${ }^{2}$ Dep. of Elect. and Electr. Eng., Bogazici University, Istanbul, Turkey
}

August 10, 2006

\begin{abstract}
Taylor's decomposition on four points is presented. Three-step difference schemes generated by the Taylor's decomposition on four points for the numerical solutions of an initial-value problem, a boundary value problem and a nonlocal boundary value problem for a third order differential equation are constructed. Numerical examples are given.
\end{abstract}

Key Words: Taylor's decomposition on four points; Third-order differential equation; Three-step difference schemes; Approximation order

AMC 2000: 65J, 65M

\section{Introduction}

In the present paper we consider the boundary-value problem of the form

$$
\left\{\begin{array}{l}
\frac{d^{3} y(t)}{d t^{3}}+a(t) y(t)=f(t), 0<t<T \\
y(0)=y_{0}, y^{\prime}(0)=y_{0}^{\prime}, y(T)=y_{T}
\end{array}\right.
$$

the initial-value problem of the form

$$
\left\{\begin{array}{l}
\frac{d^{3} y(t)}{d t^{3}}+a(t) y(t)=f(t), 0<t<T, \\
y(0)=y_{0}, y^{\prime}(0)=y_{0}^{\prime}, y^{\prime \prime}(0)=y_{0}^{\prime \prime}
\end{array}\right.
$$

and the nonlocal boundary value problem of the form

$$
\left\{\begin{array}{l}
\frac{d^{3} y(t)}{d t^{3}}+a(t) y(t)=f(t), 0<t<T \\
y(0)=y(T), y^{\prime}(0)=y^{\prime}(T), y^{\prime \prime}(0)=y^{\prime \prime}(T)
\end{array}\right.
$$

assuming $a(t)$ and $f(t)$ to be such that problems (1.1), (1.2) and (1.3) have a unique smooth solutions defined on $[0, T]$.

Modern computers allow the implementation of highly accurate difference schemes for differential equations. Hence, a task of current interest is the construction and investigation of highly accurate difference schemes for differential equations. 
In the papers [1]-[2] the two-step difference schemes generated by an exact difference scheme or by the Taylor's decomposition on two points for the numerical solutions of an initial-value problem and a boundary-value problem for a second-order differential equation are constructed. In the paper [3] the four-step difference schemes generated by the Taylor's decomposition on five points for the numerical solutions of an initial-value problem and a boundary value problem for a fourth-order differential equation are presented.

The uniform grid space

$$
[0, T]_{\tau}=\left\{t_{k}=k \tau, 0 \leq k \leq N, N \tau=T\right\}
$$

is considered for the construction of the three-step difference schemes for the approximate solutions of problems (1.1), (1.2) and (1.3). It is well known that applying the approximation of $\frac{d^{3} y\left(t_{k}\right)}{d t^{3}}$ on four points $t_{k+2}, t_{k \pm 1}, t_{k} \in[0, T]_{\tau}$ we cannot construct better than the following first order of accuracy difference scheme

$$
\left\{\begin{array}{l}
\tau^{-3}\left(u_{k+2}-3 u_{k+1}+3 u_{k}-u_{k-1}\right)+a\left(t_{k}\right) u_{k}=f\left(t_{k}\right), 1 \leq k \leq N-2, \\
u_{0}=y(0), u_{N}=y(T), \tau^{-1}\left(u_{1}-u_{0}\right)=y^{\prime}(0)
\end{array}\right.
$$

for the numerical solution of the boundary-value problem (1.1), the following first order of accuracy difference scheme

$$
\left\{\begin{array}{l}
\tau^{-3}\left(u_{k+2}-3 u_{k+1}+3 u_{k}-u_{k-1}\right)+a\left(t_{k}\right) u_{k}=f\left(t_{k}\right), 1 \leq k \leq N-2, \\
u_{0}=y(0), \tau^{-1}\left(u_{1}-u_{0}\right)=y^{\prime}(0), \\
\tau^{-2}\left(u_{0}-2 u_{1}+u_{2}\right)=y^{\prime \prime}(0)
\end{array}\right.
$$

for the numerical solution of the initial-value problem (1.2) and the following first order of accuracy difference scheme

$$
\left\{\begin{array}{l}
\tau^{-3}\left(u_{k+2}-3 u_{k+1}+3 u_{k}-u_{k-1}\right)+a\left(t_{k}\right) u_{k}=f\left(t_{k}\right), 1 \leq k \leq N-2, \\
u_{0}=u_{N},-\left(u_{N}+u_{0}\right)+\left(u_{N-1}+u_{1}\right)=0, \\
\left(u_{N}-u_{0}\right)-2\left(u_{N-1}-u_{1}\right)+\left(u_{N-2}-u_{2}\right)=0
\end{array}\right.
$$

for the numerical solution of the nonlocal boundary-value problem (1.3).

In the present paper Taylor's decomposition on four points is presented. The use of this formula gives the three-step difference schemes of fourth-order of accuracy for the approximate solutions of problems (1.1)- (1.3). Numerical examples are given.

\section{Taylor's decomposition on four points}

The utilization of Taylor's decomposition on four points in the construction of the threestep difference schemes of the fourth order of accuracy for the approximate solutions of problems (1.1) (1.2) and (1.3) is based on the following theorem. 
Theorem 2.1 Let the function $\nu(t)(0 \leq t \leq T)$ have a sixth continuous derivative and $t_{k+2}, t_{k \pm 1}, t_{k} \in[0, T]_{\tau}$. Then the following relation holds:

$$
\begin{gathered}
\tau^{-3}\left(\nu\left(t_{k+2}\right)-3 \nu\left(t_{k+1}\right)+3 \nu\left(t_{k}\right)-\nu\left(t_{k-1}\right)\right) \\
-\frac{1}{2} \nu^{\prime \prime \prime}\left(t_{k}\right)-\frac{1}{2} v^{\prime \prime \prime}\left(t_{k+1}\right)=o\left(\tau^{4}\right) .
\end{gathered}
$$

Proof. Using the Taylor's formula, we get

$$
\begin{gathered}
\tau^{-3}\left(\nu\left(t_{k+2}\right)-3 \nu\left(t_{k+1}\right)+3 \nu\left(t_{k}\right)-\nu\left(t_{k-1}\right)\right) \\
=\nu^{\prime \prime \prime}\left(t_{k}\right)+\nu^{(4)}\left(t_{k}\right) \frac{\tau}{2}+\nu^{(5)}\left(t_{k}\right) \frac{\tau^{2}}{4}+v^{(6)}\left(t_{k}\right) \frac{\tau^{3}}{12}+o\left(\tau^{4}\right) .
\end{gathered}
$$

Now, we will obtain $\alpha, \beta, \gamma$ such that

$$
\begin{gathered}
\tau^{-3}\left(\nu\left(t_{k+2}\right)-3 \nu\left(t_{k+1}\right)+3 \nu\left(t_{k}\right)-\nu\left(t_{k-1}\right)\right) \\
-\alpha \nu^{\prime \prime \prime}\left(t_{k}\right)-\beta v^{\prime \prime \prime}\left(t_{k-1}\right)-\gamma v^{\prime \prime \prime}\left(t_{k+1}\right)-d v^{\prime \prime \prime}\left(t_{k+2}\right)=o\left(\tau^{4}\right) .
\end{gathered}
$$

The use of the Taylor's formula gives

$$
\begin{gathered}
\alpha \nu^{\prime \prime \prime}\left(t_{k}\right)+\beta \nu^{\prime \prime \prime}\left(t_{k-1}\right)+\gamma \nu^{\prime \prime \prime}\left(t_{k+1}\right)+d \nu^{\prime \prime \prime}\left(t_{k+2}\right) \\
=(\alpha+\beta+\gamma+d) \nu^{\prime \prime \prime}\left(t_{k}\right)+(-\beta+\gamma+2 d) \nu^{(4)}\left(t_{k}\right) \tau \\
+\left(\frac{1}{2} \beta+\frac{1}{2} \gamma+2 d\right) \nu^{(5)}\left(t_{k}\right) \tau^{2}+\left(-\frac{1}{6} \beta+\frac{1}{6} \gamma+\frac{8}{6} d\right) v^{(6)}\left(t_{k}\right) \tau^{3}+o\left(\tau^{4}\right) .
\end{gathered}
$$

Using the last formula and formula (2.2), we obtain

$$
\begin{gathered}
\tau^{-3}\left(\nu\left(t_{k+2}\right)-3 \nu\left(t_{k+1}\right)+3 \nu\left(t_{k}\right)-\nu\left(t_{k-1}\right)\right) \\
-\alpha \nu^{\prime \prime \prime}\left(t_{k}\right)-\beta v^{\prime \prime \prime}\left(t_{k-1}\right)-\gamma v^{\prime \prime \prime}\left(t_{k+1}\right)-d v^{\prime \prime \prime}\left(t_{k+2}\right) \\
=(1-\alpha-\beta-\gamma-d) \nu^{\prime \prime \prime}\left(t_{k}\right)+\left(\frac{1}{2}+\beta-\gamma-2 d\right) \nu^{(4)}\left(t_{k}\right) \tau \\
+\left(\frac{1}{4}-\frac{1}{2} \beta-\frac{1}{2} \gamma-2 d\right) \nu^{(5)}\left(t_{k}\right) \tau^{2}+\left(\frac{1}{12}+\frac{1}{6} \beta-\frac{1}{6} \gamma-\frac{8}{6} d\right) v^{(6)}\left(t_{k}\right) \tau^{3}+o\left(\tau^{4}\right) .
\end{gathered}
$$

Then equating to zero the coefficient of the lowest power of $\tau$ in the last formula, we obtain the system of equations

$$
\left\{\begin{array}{l}
\alpha+\beta+\gamma+d=1, \\
-\beta+\gamma+2 d=\frac{1}{2} \\
\frac{1}{2} \beta+\frac{1}{2} \gamma+2 d=\frac{1}{4} \\
-\frac{1}{6} \beta+\frac{1}{6} \gamma+\frac{8}{6} d=\frac{1}{12}
\end{array}\right.
$$

From this it follows that $\alpha=\frac{1}{2}, \beta=0, \gamma=\frac{1}{2}$ and $d=0$. Theorem 2.1 is proved. 


\section{$3 \quad$ Fourth order of approximation difference scheme of the boundary-value problem (1.1)}

The construction of the three-step difference scheme of the fourth order of accuracy for the approximate solution of problem (1.1) is based on the Taylor's decomposition on four points (2.1) and on the fourth order of approximation for $v^{\prime}(0)$.

Theorem 3.1 Let the function $\nu(t)$ have a fifth continuous derivative. Then the following relation holds:

$$
\begin{gathered}
v^{\prime}(0)-\tau^{-1}\left(-\frac{3}{2} v(0)+2 v(\tau)-\frac{1}{2} v(2 \tau)\right) \\
+\tau^{2}\left\{-\frac{1}{12} v^{\prime \prime \prime}(0)-\frac{1}{4} v^{\prime \prime \prime}(\tau)\right\}=o\left(\tau^{4}\right),
\end{gathered}
$$

Proof. Using the Taylor's formula, we obtain

$$
\begin{gathered}
v^{\prime}(0)-\tau^{-1}\left(-\frac{3}{2} v(0)+2 v(\tau)-\frac{1}{2} v(2 \tau)\right) \\
-\frac{1}{3} \tau^{2} v^{\prime \prime \prime}(0)-\frac{1}{4} \tau^{3} v^{(4)}(0)=o\left(\tau^{4}\right) .
\end{gathered}
$$

Now, we will obtain $\alpha, \beta, \gamma$ such that

$$
-\frac{1}{3} \tau^{2} v^{\prime \prime \prime}(0)-\frac{1}{4} \tau^{3} v^{(4)}(0)=\tau^{2}\left[\alpha \nu^{\prime \prime \prime}(0)+\beta \nu^{\prime \prime \prime}(\tau)\right]+o\left(\tau^{4}\right) .
$$

Using the last formula and the Taylor's formula, we obtain

$$
\begin{aligned}
& -\frac{1}{3} \tau^{2} v^{\prime \prime \prime}(0)-\frac{1}{4} \tau^{3} v^{(4)}(0)-\tau^{2}\left[\alpha \nu^{\prime \prime \prime}(0)+\beta \nu^{\prime \prime \prime}(\tau)\right]+o\left(\tau^{4}\right) \\
= & \left(-\frac{1}{3}-(\alpha+\beta)\right) \tau^{2} v^{\prime \prime \prime}(0)+\left(-\frac{1}{4}-\beta\right) \tau^{3} v^{(4)}(0)+o\left(\tau^{4}\right),
\end{aligned}
$$

Then equating to zero the coefficient of the lowest power of $\tau$ in the last formula, we obtain the system of equations

$$
\left\{\begin{array}{c}
\alpha+\beta=-\frac{1}{3} \\
\beta=-\frac{1}{4} .
\end{array}\right.
$$

From that it follows $\alpha=-\frac{1}{12}$ and $\beta=-\frac{1}{4}$. Then formula (3.1) follows from this and formula (3.2). Theorem 3.1 is proved.

Applying the Taylor's decomposition on four points (2.1), formula (3.1) and equation (1.1), we obtain

$$
\begin{gathered}
\tau^{-3}\left(y\left(t_{k+2}\right)-3 y\left(t_{k+1}\right)+3 y\left(t_{k}\right)-y\left(t_{k-1}\right)\right)+\frac{1}{2} a\left(t_{k}\right) y\left(t_{k}\right)+\frac{1}{2} a\left(t_{k+1}\right) y\left(t_{k+1}\right) \\
=\frac{1}{2} f\left(t_{k}\right)+\frac{1}{2} f\left(t_{k+1}\right)+o\left(\tau^{4}\right), 1 \leq k \leq N-2 \\
y^{\prime}(0)-\tau^{-1}\left(-\frac{3}{2} y(0)+2 y(\tau)-\frac{1}{2} y(2 \tau)\right)
\end{gathered}
$$




$$
+\tau^{2}\left\{\frac{1}{12} a(0) y(0)+\frac{1}{4} a(\tau) y(\tau)\right\}+\tau^{2}\left\{-\frac{1}{12} f(0)-\frac{1}{4} f(\tau)\right\}=o\left(\tau^{4}\right),
$$

Neglecting the last small terms in the last two formulas, we obtain the fourth order of accuracy difference scheme

$$
\left\{\begin{array}{l}
\tau^{-3}\left(u_{k+2}-3 u_{k+1}+3 u_{k}-u_{k-1}\right)+\frac{1}{2} a\left(t_{k}\right) u_{k}+\frac{1}{2} a\left(t_{k+1}\right) u_{k+1} \\
=\frac{1}{2} f\left(t_{k}\right)+\frac{1}{2} f\left(t_{k+1}\right), t_{k}=k \tau, 1 \leq k \leq N-2, u_{0}=y(0), u_{N}=y(T) \\
\left(-\frac{3}{2}-\frac{1}{12} \tau^{3} a(0)\right) u_{0}+\left(2-\frac{1}{4} \tau^{3} a(\tau)\right) u_{1}-\frac{1}{2} u_{2} \\
=\tau y^{\prime}(0)+\tau^{3}\left\{-\frac{1}{12} f(0)-\frac{1}{4} f(\tau)\right\}
\end{array}\right.
$$

for the approximate solution of problem (1.1). For numerical analysis we consider the boundary-value problem

$$
\left\{\begin{array}{l}
\frac{d^{3} y(t)}{d t^{3}}+y(t)=-t^{6}+3 t^{5}-3 t^{4}-119 t^{3}+180 t^{2}-72 t+6 \\
0 \leq t \leq 1, y(0)=y^{\prime}(0)=0, y(1)=0
\end{array}\right.
$$

for the third order differential equations with the exact solution

$$
y(t)=t^{3}(1-t)^{3} .
$$

For approximate solutions of this boundary-value problem, we use the first order of accuracy difference scheme (1.4) and the fourth order of accuracy difference scheme (3.3) with different values for $\tau$, namely $\tau=\frac{1}{20}, \frac{1}{40}, \frac{1}{80}$. The errors of the numerical solutions defined as

$$
E_{N}=\max _{0 \leq k \leq N}\left|y\left(t_{k}\right)-u_{k}\right|
$$

are given in the following table.

\begin{tabular}{|l|l|l|l|}
\hline Difference schemes & $E_{20}$ & $E_{40}$ & $E_{80}$ \\
\hline Difference scheme (1.4) & 0.00160609 & 0.00073474 & 0.00035026 \\
\hline Difference scheme (3.3) & 0.00000476 & 0.00000029 & 0.00000001 \\
\hline
\end{tabular}

Thus, the fourth order of accuracy difference scheme (3.3) is more accurate comparing with the first order of accuracy difference scheme (1.4).

\section{Fourth order of approximation difference scheme of the initial-value problem (1.2)}

The construction of the three-step difference scheme of the fourth order of accuracy for the approximate solution of problem (1.2) is based on the Taylor's decomposition on four points (2.1), on the formula (3.1) and on the fourth order of approximation for $v^{\prime \prime}(0)$.

Theorem 4.1 Let the function $\nu(t)$ have a fifth continuous derivative. Then the following relations hold:

$$
\begin{gathered}
v^{\prime \prime}(0)-\tau^{-2}(v(0)-2 v(\tau)+v(2 \tau)) \\
+\tau\left\{\frac{3}{8} v^{\prime \prime \prime}(0)+\frac{2}{3} v^{\prime \prime \prime}(\tau)-\frac{1}{24} v^{\prime \prime \prime}(2 \tau)\right\}=o\left(\tau^{4}\right),
\end{gathered}
$$


Proof. Using the Taylor's formula, we obtain

$$
\begin{gathered}
v^{\prime \prime}(0)-\tau^{-2}(v(0)-2 v(\tau)+v(2 \tau)) \\
+\left\{\tau v^{\prime \prime \prime}(0)+\frac{7}{12} \tau^{2} v^{(4)}(0)+\frac{1}{4} \tau^{3} v^{(5)}(0)\right\}=o\left(\tau^{4}\right) .
\end{gathered}
$$

Now, we will obtain $\alpha, \beta, \gamma$ such that

$$
\begin{gathered}
\tau v^{\prime \prime \prime}(0)+\frac{7}{12} \tau^{2} v^{(4)}(0)+\frac{1}{4} \tau^{3} v^{(5)}(0) \\
=\tau\left[\alpha \nu^{\prime \prime \prime}(0)+\beta v^{\prime \prime \prime}(\tau)+\gamma \nu^{\prime \prime \prime}(2 \tau)\right]+o\left(\tau^{4}\right) .
\end{gathered}
$$

Using the last formula and the Taylor's formula, we obtain

$$
\begin{gathered}
\tau v^{\prime \prime \prime}(0)+\frac{7}{12} \tau^{2} v^{(4)}(0)+\frac{1}{4} \tau^{3} v^{(5)}(0) \\
-\tau\left[\alpha \nu^{\prime \prime \prime}(0)+\beta^{\prime \prime \prime}(\tau)+\gamma \nu^{\prime \prime \prime}(2 \tau)\right]+o\left(\tau^{4}\right)=(1-(\alpha+\beta+\gamma)) \tau v^{\prime \prime \prime}(0) \\
+\left(\frac{7}{12}-(\beta+2 \gamma)\right) \tau^{2} v^{(4)}(0)+\left(\frac{1}{4}-\left(\frac{1}{2} \beta+2 \gamma\right)\right) \tau^{3} v^{(5)}(0)+o\left(\tau^{4}\right) .
\end{gathered}
$$

Then equating to zero the coefficient of the lowest power of $\tau$ in the last formula, we obtain the system of equations

$$
\left\{\begin{array}{l}
\alpha+\beta+\gamma=1 \\
\beta+2 \gamma=\frac{7}{12} \\
\frac{1}{2} \beta+2 \gamma=\frac{1}{4}
\end{array}\right.
$$

Solving the last system, we get $\alpha=\frac{3}{8}, \beta=\frac{2}{3}$ and $\gamma=-\frac{1}{24}$. From this and formula (4.2) follows formula (4.1). Theorem 4.1 is proved.

Applying the Taylor's decomposition on four points (2.1), formula (4.1) and equation (1.2), we obtain

$$
\begin{gathered}
\tau^{-3}\left(y\left(t_{k+2}\right)-3 y\left(t_{k+1}\right)+3 y\left(t_{k}\right)-y\left(t_{k-1}\right)\right)+\frac{1}{2} a\left(t_{k}\right) y\left(t_{k}\right)+\frac{1}{2} a\left(t_{k+1}\right) y\left(t_{k+1}\right) \\
=\frac{1}{2} f\left(t_{k}\right)+\frac{1}{2} f\left(t_{k+1}\right)+o\left(\tau^{4}\right), 1 \leq k \leq N-2 \\
y^{\prime}(0)-\tau^{-1}\left(-\frac{3}{2} y(0)+2 y(\tau)-\frac{1}{2} y(2 \tau)\right) \\
+\tau^{2}\left\{\frac{1}{12}[a(0) y(0)-f(0)]+\frac{1}{4}[a(\tau) y(\tau)-f(\tau)]=o\left(\tau^{4}\right)\right. \\
y^{\prime \prime}(0)-\tau^{-2}(y(0)-2 y(\tau)+y(2 \tau))-\tau\left\{\frac{3}{8}[a(0) y(0)-f(0)]\right. \\
\left.\left.+\frac{2}{3}[a(\tau) y(\tau)-f(\tau)]-\frac{1}{24}[a(2 \tau) y(2 \tau)-f(2 \tau)]\right\}=o\left(\tau^{4}\right)\right\}
\end{gathered}
$$


Neglecting the last small terms in the last three formulas, we obtain the fourth order of accuracy difference scheme

$$
\left\{\begin{array}{l}
\tau^{-3}\left(u_{k+2}-3 u_{k+1}+3 u_{k}-u_{k-1}\right)+\frac{1}{2} a\left(t_{k}\right) u_{k}+\frac{1}{2} a\left(t_{k+1}\right) u_{k+1} \\
=\frac{1}{2} f\left(t_{k}\right)+\frac{1}{2} f\left(t_{k+1}\right), 1 \leq k \leq N-2, u_{0}=y(0) \\
y^{\prime}(0)-\tau^{-1}\left(-\left(\frac{3}{2}+\tau^{3} \frac{1}{12} a(0)\right) u_{0}\right. \\
\left.+\left(2-\tau^{3} \frac{1}{4} a(\tau)\right) u_{1}-\frac{1}{2} u_{2}\right)-\tau^{2}\left\{\frac{1}{12} f(0)+\frac{1}{4} f(\tau)\right\}=0 \\
y^{\prime \prime}(0)-\tau^{-2}\left(\left(1+\tau^{3} \frac{3}{8} a(0)\right) u_{0}+\left(-2+\tau^{3} \frac{2}{3} a(\tau)\right) u_{1}\right. \\
\left.+\left(1-\tau^{3} \frac{1}{24} a(2 \tau)\right) u_{2}\right)-\tau\left\{-\frac{3}{8} f(0)-\frac{2}{3} f(\tau)+\frac{1}{24} f(2 \tau)\right\}=0
\end{array}\right.
$$

for the approximate solution of problem (1.2).

For numerical analysis we consider the initial-value problem

$$
\left\{\begin{array}{l}
\frac{d^{3} y(t)}{d t^{3}}+y(t)=-t^{6}+3 t^{5}-3 t^{4}-119 t^{3}+180 t^{2}-72 t+6 \\
0 \leq t \leq 1, y(0)=y^{\prime}(0)=y^{\prime \prime}(0)=0
\end{array}\right.
$$

for the fourth order differential equations. The exact solution of this problem is:

$$
y(t)=t^{3}(1-t)^{3} .
$$

For approximate solutions of this initial-value problem, we use the first order of accuracy difference scheme (1.5) and the fourth order of accuracy difference scheme (4.3) with different values for $\tau$, namely $\tau=\frac{1}{20}, \frac{1}{40}, \frac{1}{80}$. The errors of the numerical solutions are given in the following table.

\begin{tabular}{|l|l|l|l|}
\hline Difference schemes & $E_{20}$ & $E_{40}$ & $E_{80}$ \\
\hline Difference scheme (1.5) & 0.06204958 & 0.03387875 & 0.01767174 \\
\hline Difference scheme (4.3) & 0.00000483 & 0.00000029 & 0.00000001 \\
\hline
\end{tabular}

Thus, the fourth order of accuracy difference scheme (4.3) is more accurate comparing with the first order of accuracy difference scheme (1.5).

\section{Fourth order of approximation difference scheme of the nonlocal boundary value problem (1.3)}

The construction of the three-step difference scheme of the fourth order of accuracy for the approximate solution of problem (1.3) is based on the Taylor's decomposition on four points (2.1), on the formulas (3.1), (4.1) and on the fourth order of approximation for $v^{\prime}(T)$ and $v^{\prime \prime}(T)$.

Theorem 5.1 Let the function $v(t)$ have a fifth continuous derivative. Then the following relations hold:

$$
\begin{gathered}
v^{\prime}(T)-\tau^{-1}\left(\frac{3}{2} v(T)-2 v(T-\tau)+\frac{1}{2} v(T-2 \tau)\right) \\
+\tau^{2}\left(-\frac{1}{12} v^{\prime \prime \prime}(T)-\frac{1}{4} v^{\prime \prime \prime}(T-\tau)\right)=o\left(\tau^{4}\right),
\end{gathered}
$$




$$
\begin{gathered}
v^{\prime \prime}(T)-\tau^{-2}(v(T)-2 v(T-\tau)+v(T-2 \tau)) \\
+\tau\left\{-\frac{3}{8} v^{\prime \prime \prime}(T)-\frac{2}{3} v^{\prime \prime \prime}(T-\tau)+\frac{1}{24} v^{\prime \prime \prime}(T-2 \tau)\right\}=o\left(\tau^{4}\right),
\end{gathered}
$$

Proof. First, we will prove (5.1). Using the Taylor's formula, we obtain

$$
v^{\prime}(T)-\tau^{-1}\left(\frac{3}{2} v(T)-2 v(T-\tau)+\frac{1}{2} v(T-2 \tau)\right)-\frac{1}{3} \tau^{2} v^{\prime \prime \prime}(T)+\frac{1}{4} \tau^{3} v^{(4)}(T)=o\left(\tau^{4}\right) .
$$

Now we will obtain $\alpha, \beta$ such that

$$
-\frac{1}{3} \tau^{2} v^{\prime \prime \prime}(T)+\frac{1}{4} \tau^{3} v^{(4)}(T)=\tau^{2}\left[\alpha v^{\prime \prime \prime}(T)+\beta v^{\prime \prime \prime}(T-\tau)\right]+o\left(\tau^{4}\right) .
$$

Using the last and Taylor's formula we obtain

$$
\begin{gathered}
-\frac{1}{3} \tau^{2} v^{\prime \prime \prime}(T)+\frac{1}{4} \tau^{3} v^{(4)}(T)-\tau^{2}\left[\alpha v^{\prime \prime \prime}(T)+\beta v^{\prime \prime \prime}(T-\tau)\right]+o\left(\tau^{4}\right) \\
=\left(-\frac{1}{3}-(\alpha+\beta)\right) \tau^{2} v^{\prime \prime \prime}(T)+\left(\frac{1}{4}+\beta\right) \tau^{3} v^{(4)}(T)+o\left(\tau^{4}\right)
\end{gathered}
$$

Then equating the coefficient of the lowest power of $\tau$ in the last equation we obtain the system of equations

$$
\left\{\begin{array}{c}
\alpha+\beta=-\frac{1}{3} \\
\beta=-\frac{1}{4} .
\end{array}\right.
$$

Thus we find $\alpha=-\frac{1}{12}$ and $\beta=-\frac{1}{4}$. Second, we will prove (5.2). Using the Taylor's formula, we obtain

$$
\begin{gathered}
v^{\prime \prime}(T)-\tau^{-2}(v(T)-2 v(T-\tau)+v(T-2 \tau)) \\
-\tau v^{\prime \prime \prime}(T)+\frac{7}{12} \tau^{2} v^{(4)}(T)-\frac{1}{4} \tau^{3} v^{(5)}(T)=o\left(\tau^{4}\right),
\end{gathered}
$$

Now,we will obtain $\alpha, \beta$ and $\gamma$ such that

$$
-\tau v^{\prime \prime \prime}(T)+\frac{7}{12} \tau^{2} v^{(4)}(T)-\frac{1}{4} \tau^{3} v^{(5)}(T)=\tau\left\{\alpha v^{\prime \prime \prime}(T)+\beta v^{\prime \prime \prime}(T-\tau)+\gamma v^{\prime \prime \prime}(T-2 \tau)\right\}+o\left(\tau^{4}\right),
$$

Using the last and Taylor's formula we obtain

$$
\begin{aligned}
& -\tau v^{\prime \prime \prime}(T)+\frac{7}{12} \tau^{2} v^{(4)}(T)-\frac{1}{4} \tau^{3} v^{(5)}(T)-\tau\left\{\alpha v^{\prime \prime \prime}(T)+\beta v^{\prime \prime \prime}(T-\tau)+\gamma v^{\prime \prime \prime}(T-2 \tau)\right\}+o\left(\tau^{4}\right) \\
& =(-1-(\alpha+\beta+\gamma)) \tau v^{\prime \prime \prime}(T)+\left(\frac{7}{12}-(-\beta-2 \gamma)\right) \tau^{2} v^{(4)}(T)+\left(-\frac{1}{4}-\left(\frac{1}{2} \beta+2 \gamma\right)\right) v^{(5)}(T)+o\left(\tau^{4}\right),
\end{aligned}
$$

Then equating the coefficients of the lowest power of $\tau$ in the last equation we obtain the system of equations 


$$
\left\{\begin{array}{l}
\alpha+\beta+\gamma=-1 \\
-\beta-2 \gamma=\frac{7}{12} \\
\frac{1}{2} \beta+2 \gamma=-\frac{1}{4}
\end{array}\right.
$$

From that it follows $\alpha=-\frac{3}{8}, \beta=-\frac{2}{3}$, and $\gamma=\frac{1}{24}$. Theorem 5.2 is proved.

Applying the Taylor's decompositim on four points,formulas (5.1), (5.2) and equation (3.1), we obtain

$$
\begin{gathered}
\tau^{-3}\left(y\left(t_{k+2}\right)-3 y\left(t_{k+1}\right)+3 y\left(t_{k}\right)-y\left(t_{k-1}\right)\right)+\frac{1}{2} a\left(t_{k}\right) y\left(t_{k}\right)+\frac{1}{2} a\left(t_{k+1}\right) y\left(t_{k+1}\right) \\
=\frac{1}{2} f\left(t_{k}\right)+\frac{1}{2} f\left(t_{k+1}\right)+o\left(\tau^{4}\right), 1 \leq k \leq N-2 \\
\left(\frac{3}{2} y(T)-2 y(T-\tau)+\frac{1}{2} y(T-2 \tau)\right)+\tau^{3}\left(\frac{1}{12} y^{\prime \prime \prime}(T)+\frac{1}{4} y^{\prime \prime \prime}(T-\tau)\right) \\
-\left(-\frac{3}{2} y(0)+2 y(\tau)-\frac{1}{2} y(2 \tau)\right)-\tau^{3}\left(\frac{1}{12} y^{\prime \prime \prime}(0)+\frac{1}{4} y^{\prime \prime \prime}(\tau)\right)=o\left(\tau^{4}\right) \\
(y(T)-2 y(T-\tau)+y(T-2 \tau))+\tau^{3}\left\{\frac{3}{8} y^{\prime \prime \prime}(T)+\frac{2}{3} y^{\prime \prime \prime}(T-\tau)-\frac{1}{24} y^{\prime \prime \prime}(T-2 \tau)\right\} \\
-(y(0)-2 y(\tau)+y(2 \tau))-\tau^{3}\left\{-\frac{3}{8} y^{\prime \prime \prime}(0)-\frac{2}{3} y^{\prime \prime \prime}(\tau)+\frac{1}{24} y^{\prime \prime \prime}(2 \tau)\right\}=o\left(\tau^{4}\right),
\end{gathered}
$$

Neglecting the last small terms in the last three formulas, we obtain the fourth order of accuracy difference scheme

$$
\left\{\begin{array}{l}
\tau^{-3}\left(u_{k+2}-3 u_{k+1}+3 u_{k}-u_{k-1}\right)+\frac{1}{2} a\left(t_{k}\right) u_{k}+\frac{1}{2} a\left(t_{k+1}\right) u_{k+1} \\
=\frac{1}{2} f\left(t_{k}\right)+\frac{1}{2} f\left(t_{k+1}\right), 1 \leq k \leq N-2, u_{0}=u_{N} \\
\left(-\frac{3}{2}-\frac{1}{12} \tau^{3} a(0)\right) u_{0}+\left(2-\frac{1}{4} \tau^{3} a(\tau)\right) u_{1}-\frac{1}{2} u_{2} \\
-\left(\frac{3}{2}-\frac{1}{12} \tau^{3} a(T)\right) u_{N}-\left(-2-\frac{1}{4} \tau^{3} a(T-\tau)\right) u_{N-1}-\frac{1}{2} u_{N-2} \\
=\frac{1}{12} \tau^{3}(f(T)-f(0))+\frac{1}{4} \tau^{3}(f(T-\tau)-f(\tau)) \\
\left(1+\frac{3}{8} \tau^{3} a(0)\right) u_{0}+\left(-2+\frac{2}{3} \tau^{3} a(\tau)\right) u_{1}+\left(1-\frac{1}{24} \tau^{3} a(2 \tau)\right) u_{2} \\
-\left(1-\frac{3}{8} \tau^{3} a(T)\right) u_{N}-\left(-2-\frac{2}{3} \tau^{3} a(T-\tau)\right) u_{N-1} \\
-\left(1+\frac{1}{24} \tau^{3} a(T-2 \tau)\right) u_{N-2}=\tau^{3}\left\{\frac{3}{8}(f(T)+f(0))\right. \\
\left.+\frac{2}{3}(f(T-\tau)+f(\tau))-\frac{1}{24}(f(T-2 \tau)+f(2 \tau))\right\}
\end{array}\right.
$$

for the approximate solution of problem (1.3) .

Now, for numerical analysis we consider the nonlocal boundary value problem

$$
\left\{\begin{array}{l}
\frac{d^{3} y(t)}{d t^{3}}+y(t)=-t^{6}+3 t^{5}-3 t^{4}-119 t^{3}+180 t^{2}-72 t+6 \\
0 \leq t \leq 1, y(0)=y(1), y^{\prime}(0)=y^{\prime}(1), y^{\prime \prime}(0)=y^{\prime \prime}(1)
\end{array}\right.
$$

for the third order differential equations. The exact solution of this problem is

$$
y(t)=t^{3}(1-t)^{3} .
$$

For approximate solutions of this nonlocal boundary-value problem, we use the first order of accuracy difference scheme (1.6) and the fourth order of accuracy difference scheme 
(5.3) with different values for $\tau$, namely $\tau=\frac{1}{20}, \frac{1}{40}, \frac{1}{80}$. The errors of the numerical solutions are defined as

$$
E_{N}=\max _{0 \leq k \leq N}\left|y\left(t_{k}\right)-u_{k}\right| \text {. }
$$

\begin{tabular}{|l|l|l|l|}
\hline Difference schemes & $E_{20}$ & $E_{40}$ & $E_{80}$ \\
\hline Difference scheme (1.6) & 0.16010542 & 0.11498498 & 0.06658580 \\
\hline Difference scheme (5.3) & 0.00000312 & 0.00000019 & 0.00000001 \\
\hline
\end{tabular}

Hence, the fourth order of accuracy difference scheme (5.3) is more accurate when compared with the first order of accuracy difference scheme (1.6).

It is known that various boundary value problems for the partial differential equations can be reduced to the boundary value problem

$$
\left\{\begin{array}{c}
\frac{d^{3} y(t)}{d t^{3}}+A(t) y(t)=f(t), 0<t<T \\
y(0)=y_{0}, y^{\prime}(0)=y_{0}^{\prime}, y(T)=y_{T}
\end{array}\right.
$$

and the initial-value problem

$$
\left\{\begin{array}{l}
\frac{d^{3} y(t)}{d t^{3}}+A(t) y(t)=f(t), 0<t<T \\
y(0)=y_{0}, y^{\prime}(0)=y_{0}^{\prime}, y^{\prime \prime}(0)=y_{0}^{\prime \prime}
\end{array}\right.
$$

and the nonlocal boundary value problem

$$
\left\{\begin{array}{l}
\frac{d^{3} y(t)}{d t^{3}}+A(t) y(t)=f(t), 0<t<T \\
y(0)=y(T), y^{\prime}(0)=y^{\prime}(T), y^{\prime \prime}(0)=y^{\prime \prime}(T)
\end{array}\right.
$$

for the differential equations in a Hilbert space $H$, with the self-adjoint positive definite operators $A(t)$. The use of Taylor's decomposition on four points permits to construct the difference schemes of the fourth order of accuracy for the approximate solutions of problems (5.4)-(5.6). Operator method of [4] permits to establish the stability of these difference schemes.

\section{References}

[1] A. Ashyralyev, P.E. Sobolevskii, On the two new approaches for construction of the high order of accuracy difference schemes for the second order differential equations, Functional Differential Equations, 10(3-4)(2003), 333-405.

[2] A. Ashyralyev, P.E. Sobolevskii, On the two-step the high order of accuracy difference schemes for the second order differential equations, Proceedings of Dynamic Systems and Applications, 4(2004), 528-535.

[3] M.A. Ashyralyeva, P.E. Sobolevskii, A note on the Taylor's decomposition on five points and its applications to differential equations, Functional Differential Equations, $13(3-4)(2006)$.

[4] A. Ashyralyev, P.E. Sobolevskii, New Difference Schemes for Partial Differential Equations. Birkhauser Verlag: Basel, Boston, Berlin. 2004. 
[5] V.I. Gorbachuk, M.L. Gorbachuk, Boundary Value Problems for Differential-Operator Equations, Naukova Dumka: Kiev (1984).(Russian).

[6] T.D. Dzhuraev, Boundary Value Problems for Equations of Mixed and MixedComposite Types, Fan:Tashkent, 1979. (Russian).

[7] D. Bazarov, H. Soltanov, Some Local and Nonlocal Boundary Value Problems for Equations of Mixed and Mixed-Composite Types, Ylim: Ashgabat, 1995. (Russian).

[8] A.G. Latrous, A. Memou, A three-point boundary value problem with an integral condition for a third-order partial differential equation, Abstract and Applied Analysis, 2005 (1)(2005), 33-43. 